\title{
Emphysematous pyelonephritis in a patient with polycystic kidney disease
}

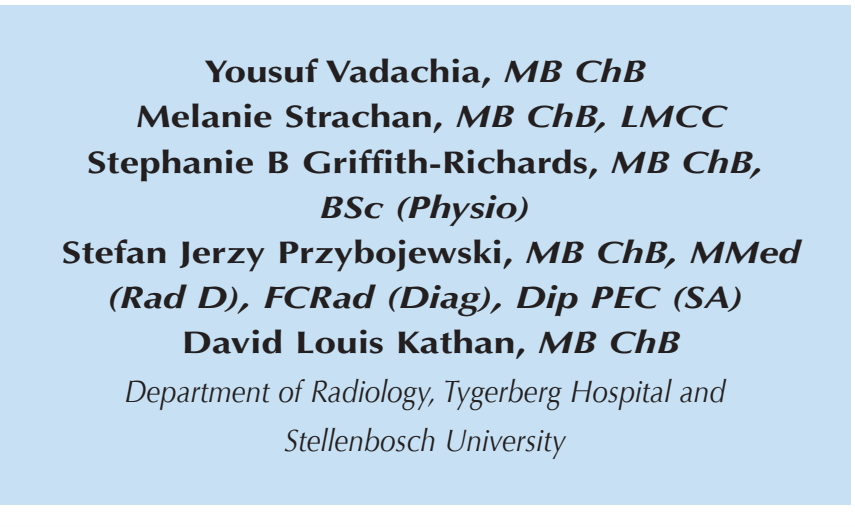

\section{Abstract}

A case report demonstrating the plain film and CT findings of emphysematous pyelonephritis in a patient with polycystic kidney disease, an extremely rare occurrence.

\section{Introduction}

Emphysematous pyelonephritis is a rare, complicated pyelonephritis that is considered life-threatening. ${ }^{1,2}$ It is extremely rare in the literature in combination with autosomal dominant polycystic kidney disease. We present such a case with pertinent diagnostic imaging.

\section{Case report}

\section{Clinical details}

A 36-year-old female patient, known with type 1 diabetes mellitus was referred to our hospital with a 2-day history of frank haematuria, severe lower back ache, diarrhoea and vomiting. The patient had been feeling unwell for a few days prior to the haematuria episode. She was known to have autosomal dominant polycystic kidney disease with mild chronic renal impairment and hypertension.

On physical examination, the patient was pyrexial with a temperature of 38 degrees Celsius. She was tachycardic, with a pulse of $110 \mathrm{bpm}$, and hypotensive with a blood pressure of $96 / 54 \mathrm{mmHg}$. The patient had severe left-sided renal angle tenderness. The rest of the abdomen was soft, with no signs of peritonism.

The patient's urine showed macroscopic haematuria and leucocytes were present on dipstick testing. The blood urea at the time was $22.4 \mathrm{mmol} / \mathrm{l}$; the serum creatinine was $284 \mu \mathrm{mol} / \mathrm{l}$.

\section{Imaging findings}

Plain radiographs of the chest and abdomen showed an elevated left hemidiaphragm, a medially displaced stomach bubble and a large, ovoid gas shadow in the left flank region (Figs 1 and 2). An ultrasound of the kidneys was requested which showed the typical appearance of polycystic kidney disease on the right side. The left kidney was obscured by gas artefact and visualisation of the spleen, the left renal bed and the psoas region was very poor.

In the light of the patient's severe illness, pyrexia and renal impairment, a CT scan of the abdomen was performed. The patient took oral contrast material prior to the scan. No intravenous contrast was used. The CT scan showed multiple cysts in both kidneys with the left kidney more severely involved than the right. There were small gas locules outlining the margin of the left kidney. A large, laterally positioned cyst contained mainly gas with a prominent gas/fluid level. A second large, medially positioned cyst contained fluid

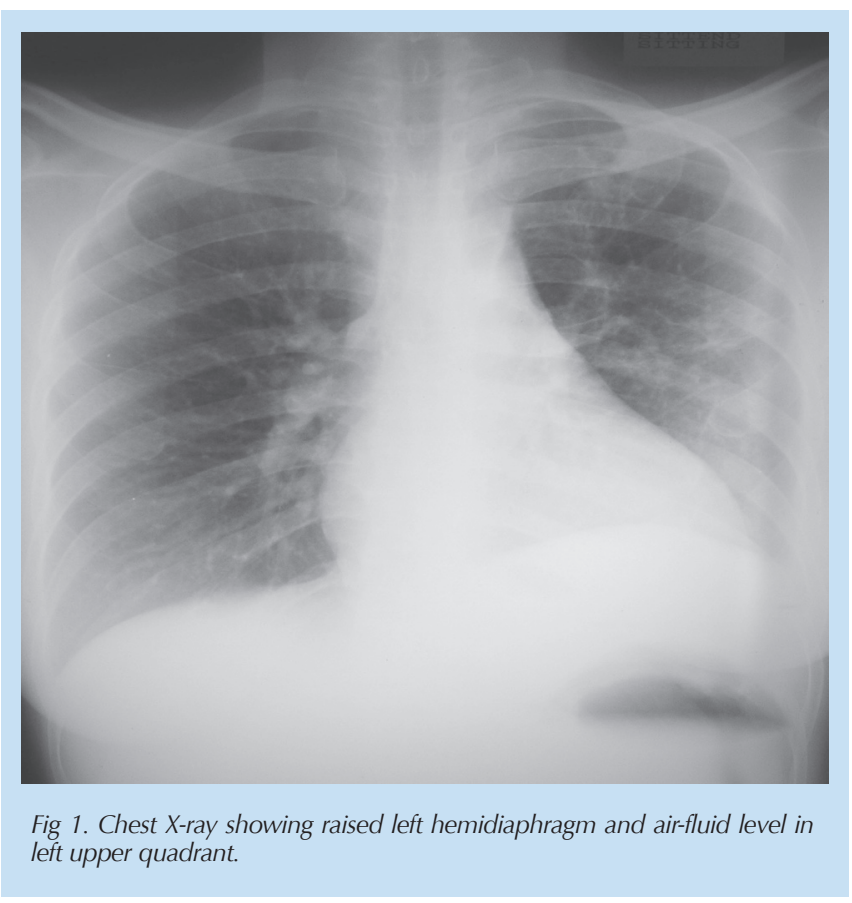




\section{CASE REPORT}

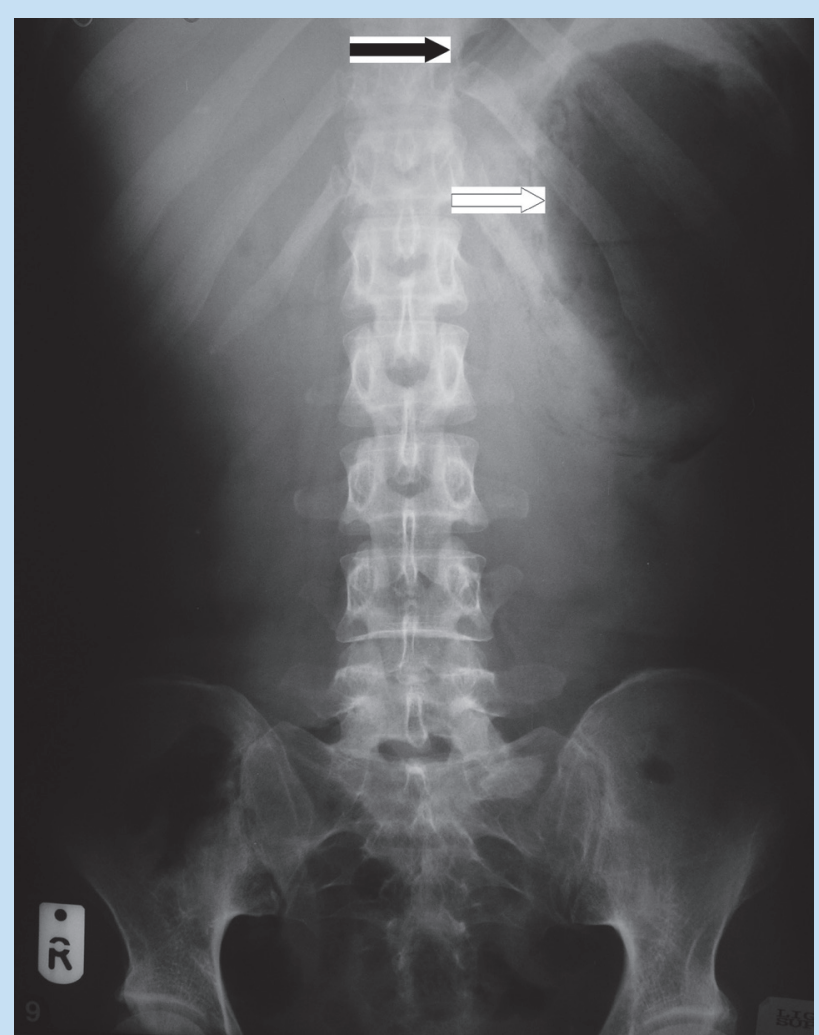

Fig. 2. Abdominal X-Ray, showing the displaced stomach bubble (black arrow). Laterally, a large ovoid gas shadow is seen (white arrow).

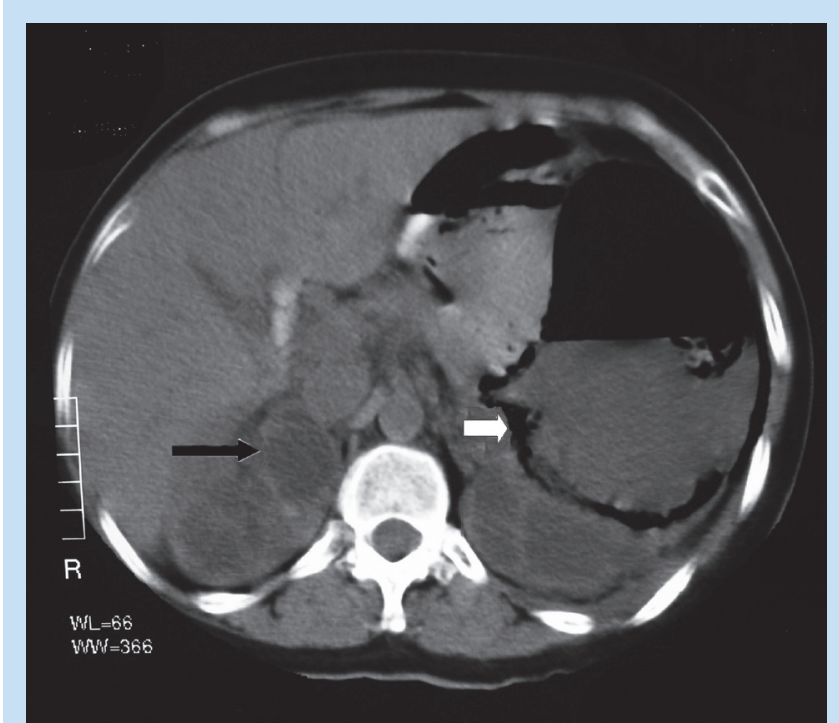

Fig. 3. CT scan at the level of porta hepatis. Multiple renal cysts are seen (black arrow). A large air-fluid level is seen in a laterally positioned left renal cyst as well as gas outlining the wall of the cyst (white arrow).

that appeared denser than the remaining cysts, as well as small locules of gas (Fig. 3). There was marked fat stranding around the left kidney. The contrast-filled stomach was medially displaced (Fig. 4). There was no evidence of free air within the peritoneal cavity.

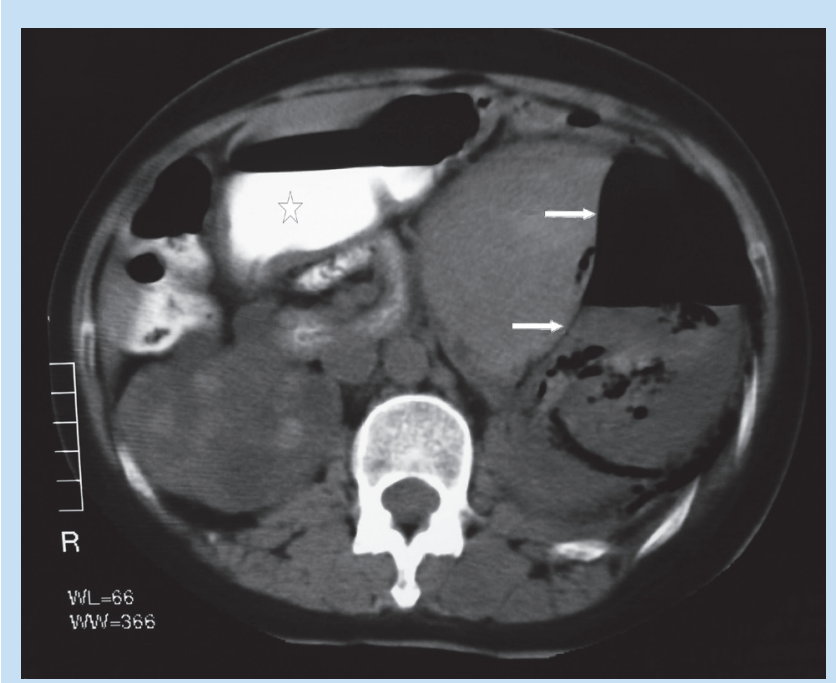

Fig. 4. CT scan at the level of the lower pole of both kidneys. The contrastfilled stomach is displaced medially (star). A thin membrane is seen (white arrow) separating two large cysts in the left kidney. The lateral cyst is filled with necrotic slough, pus and gas, the medial cyst is filled with denser material with small locules of gas. These were the 2 cysts confirmed to be infected at the time of surgery.

\section{Clinical outcome}

The patient was taken to emergency surgery. The left renal bed was explored and 2 large gas-containing cysts were found. There was a large amount of necrotic slough within the cysts, as well as in the adjacent renal tissue. The cysts were drained, some debridement of the surrounding tissue was done and surgical drains were left in situ.

The patient recovered slowly with drainage and antibiotic cover. Her renal function slowly improved to her baseline level of functioning, with a blood urea of 13.3 $\mathrm{mmol} / \mathrm{l}$ and serum creatinine of $160 \mu \mathrm{mol} / \mathrm{l}$. Microscopy of tissue removed at the time of the surgery showed necrotic material with numerous Gram-negative bacilli. Culture yielded Escherichia coli.

\section{Discussion}

Emphysematous pyelonephritis is a rare and life-threatening condition. Prompt diagnosis allows for appropriate management to be initiated speedily. Clinical findings are often non-specific and radiographic confirmation of gas within the kidney is diagnostic. ${ }^{3}$ Our 


\section{CASE REPORT}

case demonstrates some of the radiological signs in the extremely rare occurrence of emphysematous pyelonephritis in a polycystic kidney.

Plain film. The gas filling the renal cyst and renal parenchyma appears as a lucent cavity in the region of the left renal bed. Gas collection in the renal parenchyma may appear as mottled radiolucent shadows on the plain film. ${ }^{1}$ The raised left hemidiaphragm in our patient may represent a large subphrenic inflammatory mass, or may be due to the enlarged polycystic left kidney.

Ultrasound. Chou et al. ${ }^{1}$ highlight a few ultrasound findings which may be helpful in diagnosing emphysematous pyelonephritis, such as echogenic gas locules within the renal parenchyma, and 'dirty shadows' ${ }^{1}$ cast by gas locules. Owing to the gas outlining the left kidney in our patient, visualising the renal parenchyma was extremely difficult. Furthermore, the normal renal architecture was distorted by the polycystic disease. Thus ultrasound was not helpful in the diagnosis of this case.

CT scanning. CT scan is considered to be the best modality for confirmation of the diagnosis. ${ }^{2}$ This was borne out in our case. The gas within the involved renal cysts was clearly visualised, as well as gas outlining the kidney within Gerota's fascia. The inflammatory changes and fat stranding around the left kidney was visualised. The denser fluid within the medial large cyst is most likely purulent fluid and/or blood.

The aetiology of emphysematous pyelonephritis has been postulated to be due to fermentation of glucose by gas-producing organisms, especially $E$. coli, in diabetics to form carbon dioxide and hydrogen. ${ }^{2}$ Anaerobic organisms are rare pathogens. ${ }^{2}$ In non-diabetics the presence of impaired host immunity, focal renal infarctions, or obstruction are considered to be predisposing factors. ${ }^{4}$ Our patient appears to be at higher than normal risk due to the combination of diabetes and adult polycystic kidney disease. Haemorrhage into the cysts is a known complication of polycystic kidney disease. This could conceivably have been the precipitating factor causing obstruction of the involved cysts, colonisation by gas-forming bacteria and a rich substrate for bacterial growth leading to the emphysematous pyelonephritis.

1. Chou YH, Tiu CM, Chen TW, Chen KK, Chang T. Emphysematous pyelonephritis in a polycystic kidney Demonstration by ultrasound and computed tomography J Ultrasound Med 1990; 9: 355-357.

2. Shokeir AA, El-Azab M, Mohsen T, El-Diasty T. Emphysematous pyelonephritis: a 15 year experience with 20 cases. Urology 1997; 49: 343-346.

3. Evanoff GV, Thompson CS, Foley R, Weinman EJ. Spectrum of gas within the kidney. Emphysematous pyelonephritis and emphysematous pyelitis. Am J Med 1987; 83: 149-154.

4. Levinson ED, Weidner FA. Emphysematous pyelonephritis in a polycystic kidney. Urol Radiol 1981; 3 39-41. 